\title{
PETER Hilton
}

\section{Localization and cohomology of nilpotent groups}

Cahiers de topologie et géométrie différentielle catégoriques, tome 14, no 4 (1973), p. 341-369

<http://www.numdam.org/item?id=CTGDC_1973_14_4_341_0>

(C) Andrée C. Ehresmann et les auteurs, 1973, tous droits réservés.

L'accès aux archives de la revue «Cahiers de topologie et géométrie différentielle catégoriques » implique l'accord avec les conditions générales d'utilisation (http://www.numdam.org/conditions). Toute utilisation commerciale ou impression systématique est constitutive d'une infraction pénale. Toute copie ou impression de ce fichier doit contenir la présente mention de copyright.

\section{Numdam}




\title{
LOCALIZATION AND COHOMOLOGY OF NILPOTENT GROUPS *
}

\author{
by Peter HILTON
}

\section{Introduction.}

The theory of the $P$-localization of groups, where $P$ is a family of primes, appears to have been first discussed by Malcev and Lazard $[9,10]$. In their work emphasis was placed on the explicit construction of the localization and properties of the localization $G_{P}$ of the nilpotent group $G$ were deduced from the construction, utilizing nilpotent group theory. Baumslag [1] has given a comprehensive treatment of the main properties of nilpotent groups as they relate to the problem of localization, and has himself [2] explicitly shown how to construct $G_{P}$ in the case of an arbitrary nilpotent group $G$ and an arbitrary family of primes $P$, thus extending the generality of Malcev's original construction. Bousfield-Kan [3] exploit this general Malcev construction in their study of completion and localization. There is a very readable account of the theory of nilpotent groups and a description of the localization construction in the notes of Warfield [ 11 ].

In this paper we adopt a completely different approach. Starting with the completely elementary theory of localization of abelian groups, we show that a localizing functor $L: N_{c} \rightarrow N_{c}$ may be built up inductively with respect to $c$, where $N_{c}$ is the category of nilpotent groups of nilpotency class $\leqslant c$. Our tool in demonstrating this is the cohomology theory of groups and, in particular, the interpretation of $H^{2}(Q ; A)$, where $A$ is an abelian group (trivial $Q$-module), as the collection of equivalence classes of central extensions of $Q$ by the abelian central kernel $A$. We incorporate into the inductive hypothesis the key fact that the natural (localizing) homomorphism $e: G \rightarrow G_{P}$ (where $G_{P}=L G$ ) is a $P$-isomorphism, meaning that kere consists of elements of torsion prime to $P$ and that, for any $y \in G_{P}$, there exists $n$ prime to $P$ with $y^{n} \in i m e$.

* Conférence donnée au Colloque d'Amiens. 
In our approach we do not have to construct $G_{P}$ explicitly; we simply prove that it exists and that the functor $L$ and the natural transformation $e$ have certain important properties. By using this approach we are able to deduce various properties of nilpotent groups, some of which make no explicit mention of localization.

The plan of the paper is as follows. In Section 2 we document the facts we need about the localization of abelian groups - essentially to start the induction. In Section 3 we describe the (co)homology theory of groups, especially with regard to the second cohomology group, and we recall the Lyndon-Hochschild-Serre spectral sequence. In Section 4 we prove the main results, showing that the localization exists and has properties which we demand of it; as indicated, a key property is already built into the inductive hypothesis. In Section 5 we make applications to the theory of nilpotent groups; we emphasize that no sophisticated theory has gone into these applications; we simply exploit the fact that localization exists and has some nice properties.

Among the facts which immediately emerge is that if $\phi: G \rightarrow H$ is a $P$-isomorphism of nilpotent groups, then $\phi_{P}: G_{P} \rightarrow H_{P}$ is an isomorphism. To prove the converse of this appears to require deeper properties of nilpotent groups than those exploited in Sections 2-5 - indeed, in those sections we use nothing deeper than a group of nilpotency class $c$ is naturally expressible as a central extension of a group of nilpotency class $c-1$. However, in Section 6, we refer to Hall's theory of basic commutators [5] in order to prove a lemma which turns out to be crucial in establishing the converse. Once established, the converse then enables us to develop a theory of $P$-isomorphisms of nilpotent groups which parallels standard results on nilpotent groups.

In Section 7 we prove various results on the localization of nilpotent groups which are particularly revelant to the study of nilpotent spaces $[6,7]$.

The author wishes to acknowledge very valuable conversations with Guido Mislin, Joe Roitberg, and Urs Stammbach. A proof of a weaker form of Theorem 6.1, perfectly adequate for the proof of Corollary 6.4, was communicated to the author by Bill Waterhouse. 


\section{Localization of abelian groups.}

In this section we collect together the results we will need on the $P$-localization $e: A \rightarrow A_{P}$ of an abelian group, with respect to a family of primes $P$. We recall that $A_{P}=A \otimes \mathbf{Z}_{P}$, where $\mathbf{Z}_{P}$ is the ring of integers localized at $P$, that is, the subring of the rationals $Q$, consisting of those rationals $k / l$ such that $l$ is prime to $P$; and $e$ is the natural homomorphism $a \rightarrow a \otimes 1$. Note that a $P$-local group is just a $\mathbf{Z}_{P}$-module.

PROPOSITION 2.1. Localization is exact.

PROPOSITION 2.2. Localization commutes with direct limits.

PROPOSITION 2.3. Given

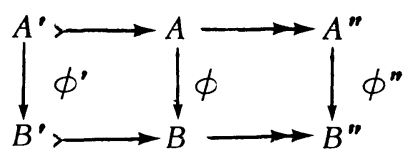

with exact rows, then if any two of $\phi^{\prime}, \phi, \phi^{\prime \prime} P$-localize, so does the third.

PROPOSITION 2.4.

$$
\begin{gathered}
e \otimes 1: A \otimes B \rightarrow A_{P} \otimes B, \quad e \otimes e: A \otimes B \rightarrow A_{P} \otimes B_{P} \\
\operatorname{Tor}(e, 1): \operatorname{Tor}(A, B) \rightarrow \operatorname{Tor}\left(A_{P}, B\right) \\
\operatorname{Tor}(e, e): \operatorname{Tor}(A, B) \rightarrow \operatorname{Tor}\left(A_{P}, B_{P}\right)
\end{gathered}
$$

all P-localize.

PROOF. The first two assertions are obvious; we will be content to prove the third. Let $R \mapsto F \rightarrow A$ be a free abelian presentation of $A$. Then $R_{P} \rightarrow F_{P} \rightarrow A_{P}$ is exact with $R_{P}, F_{P}$ flat. Thus we have

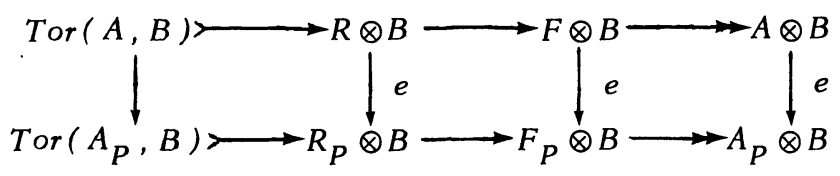

Two applications of Proposition 2.3 now show that $\operatorname{Tor}(e, 1) P$-localizes.

PROPOSITION 2.5. $\widetilde{H}_{*}(e): \widetilde{H}_{*}(A) \rightarrow \widetilde{H}_{*}\left(A_{P}\right)$ P-localizes, where $\widetilde{H}_{*}$ is 
reduced bomology with integer coefficients.

PROOF. Let $\mathcal{F}$ be the family of abelian groups for which the assertion holds. First, we claim that $\mathbf{Z} / p^{k} \in \mathcal{F}$. For if $A=\mathbf{Z} / p^{k}, p \in P$, then $A_{P}=A, e=1$, and $\widetilde{H}_{*}(A)$ is $P$-local, so the assertion is clear. If $A=\mathbf{Z} / p^{k}, p \in P^{\prime}$, the complementary set of primes, then $A_{P}=0, \widetilde{H}_{*}(A)$ is $P^{\prime}$-torsion, and the assertion is again clear.

Second, we claim that $\mathbf{Z} \in \mathcal{F}$. For $\mathbf{Z}_{P}$ is torsion-free of rank 1 , so that

$$
H_{n}\left(\mathbf{Z}_{P}\right)=\mathbf{Z}_{P}, \quad n=1, \quad H_{n}\left(\mathbf{Z}_{P}\right)=0, n \geqslant 2 .
$$

Thus $\widetilde{H}_{*}(e)=H_{1}(e)$ and $H_{1}(e)$ plainly $P$-localizes.

We next argue that, if $A, B \in \mathcal{F}$, so does $A \oplus B$. This follows immediately from the natural Künneth formula for $H_{*}(A \oplus B)$, together with Propositions 2.3 and 2.4 . Thus we have proved that if $A$ is finitely generated, then $A \in \mathcal{F}$.

Finally, we use a direct limit argument to show that every $A \in \mathcal{F}$. For $A$ is the (directed) union of its finitely generated subgroups, $H_{*}$ commutes with direct limits, and Proposition 2.2 then enables us to complete the argument.

DEfinition. We say that $\phi: A \rightarrow B$ is a $P$-isomorpbism if ker $\phi$, coker $\phi$ are both $P^{\prime}$-torsion groups.

PROPOSITION 2.6. $\phi: A \rightarrow B \quad P$-localizes if and only if $B$ is $P$-local and $\phi$ is a $P$-isomorphism.

P R OOF. We first show that $e: A \rightarrow A_{P}$ is a $P$-isomorphism. We may embed $e$ in the exact sequence

$$
\operatorname{Tor}\left(A, \mathbf{Z}_{P} / \mathbf{Z}\right) \longrightarrow A \stackrel{e}{\longrightarrow} A \longrightarrow \mathbf{Z}_{P} / \mathbf{Z} \text {. }
$$

Thus we must show that $\operatorname{Tor}\left(A, \mathbf{Z}_{P} / \mathbf{Z}\right)$ and $A \otimes \mathbf{Z}_{P} / \mathbf{Z}$ are $P^{\prime}$-torsion groups. Now

$$
\mathbf{Z}_{P} / \mathbf{Z}=\underset{p \in P^{\oplus}}{\oplus} \mathbf{Z} / p^{\infty},
$$

where $\mathbf{Z} / p^{\infty}$ is the $p$-Prüfer group. It is thus plain that $Z_{p} / \mathbf{Z}$ is a $P^{\prime}$-torsion group, from which it readily follows that $A \otimes \mathbf{Z}_{P} / \mathbf{Z}$ and 
$\operatorname{Tor}\left(A, \mathbf{Z}_{P} / \mathbf{Z}\right)$ are $P^{\prime}$-torsion groups.

Conservely suppose that $B$ is $P$-local and $\phi: A \rightarrow B$ is a $P$-isomorphism. We thus have a commutative diagram

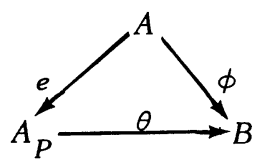

and the proof of Proposition 2.6 is completed by means of the following two lemmas.

LEMMA 2.7. If $\beta \alpha$ and $\alpha$ are $P$-isomorphisms, so is $\beta$.

LEMMA 2.8. If $\theta: C \rightarrow D$ is a $P$-isomorphism and $C, D$ are $P$-local, then $\theta$ is an isomorphism.

PROOF of 2.7. It is trivial that if $\operatorname{coker} \beta \alpha$ is $P^{\prime}$-torsion, so is coker $\beta$. From the exact sequence

$$
\operatorname{ker} \alpha \longrightarrow \operatorname{ker} \beta \alpha \longrightarrow \operatorname{ker} \beta \longrightarrow \operatorname{coker} \alpha
$$

we deduce that if $\operatorname{ker} \beta \alpha$ and coker $\alpha$ are $P^{\prime}$-torsion, so is ker $\beta$.

P R OOF of 2.8. It is trivial that if ker $\theta$ is $P^{\prime}$-torsion and $C$ is $P$-local, then $\operatorname{ker} \theta=0$. Now suppose $\operatorname{coker} \theta$ is $P^{\prime}$-torsion and let $d \in D$. Then $n d=\theta c$ for some $c \in C, n \in P^{\prime}$. Since $C$ is $P$-local, $c=n c_{1}, c_{1} \in C$, so $n\left(d-\theta c_{1}\right)=0$. Since $D$ is $P$-local, $d-\theta c_{1}=0$, so $\operatorname{coker} \theta=0$.

PRoposition 2.9. If $B$ is $P$-local, then $e^{*}: \operatorname{Hom}\left(A_{P}, B\right) \cong \operatorname{Hom}(A, B)$, $e^{*}: \operatorname{Ext}\left(A_{P}, B\right) \cong E x t(A, B)$.

PROOF. The first isomorphism simply expresses the universal property of $e$. As to the second, let $B \mapsto I \rightarrow J$ be an injective presentation of $B$ as $\mathbf{Z}_{P}$-module. Since $\mathbf{Z}_{P}$ is flat, it follows that $B \mapsto I \rightarrow J$ is also an injective presentation of $B$ as abelian group. Thus we have a commutative diagram

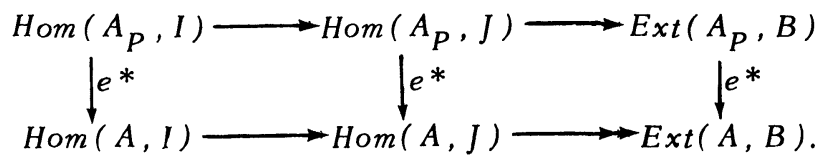

Since the first two vertical arrows are isomorphisms, so is the third. 


\section{Cohomology and central extensions.}

In this section we recall the results we will need on the homology and cohomology of groups. We will be concerned exclusively with cohomology groups $H^{k}(Q ; A)$, where $A$ is a trivial $Q$-module.

PROPOSITION 3.1. There is a natural universal coefficient sequence

$$
\operatorname{Ext}\left(H_{k-1} Q, A\right) \longmapsto H^{k}(Q ; A) \longrightarrow H o m\left(H_{k} Q, A\right) \text {. }
$$

We now study the interpretation of $H^{2}(Q ; A)$ as the group of equivalence classes of central extensions of the quotient group $Q$ by the abelian kernel $A$. Let $\xi \in H^{2}(Q ; A)$ be represented by

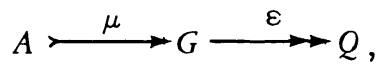

and let $\phi: A \rightarrow A_{1}$. Form the product $A_{1} \times G$ and embed $A$ by $\left(^{(*)}\right.$ $a \rightarrow(-\phi a, \mu a)$. Then $A$ is central in $A_{1} \times G$ and we may form the quotient group $G_{1}$; write an element of $G_{1}$ as $\left\{a_{1}, g\right\}$, the coset containing $\left(a_{1}, g\right)$. There are evident maps $\mu_{1}: A_{1} \rightarrow G_{1}, \psi: G \rightarrow G_{1}$, given by $\mu_{1}\left(a_{1}\right)=\left\{a_{1}, 1\right\}, \psi(g)=\{0, g\}$ and

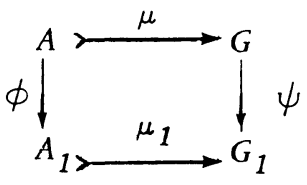

commutes. Moreover

PROPOSITION 3.2. In (3.2), $\mu_{1}$ maps $A_{1}$ injectively to a central subgroup of $G_{1}$ and coker $\mu_{1}=Q$.

PROOF. It is trivial that $\mu_{1}$ is injective and that $\mu_{1} A_{1}$ is central in $G_{1}$. Let $\varepsilon_{1}: G_{1} \rightarrow Q$ be given by $\varepsilon_{1}\left\{a_{1}, g\right\}=\varepsilon g$. It is plain that $\varepsilon_{1}$ is welldefined and surjective. Moreover $\varepsilon_{1} \mu_{1}=0$, and, if $\varepsilon_{1}\left\{a_{1}, g\right\}=1$, then $g=\mu a$, so

$$
\left\{a_{1}, g\right\}=\left\{a_{1}, \mu a\right\}=\left\{a_{1}+\phi a, 1\right\}=\mu_{1}\left(a_{1}+\phi a\right) .
$$

Thus (3.2) enlarges to a map of central extensions

(*) Note that $A$ is written additively, while $G$ and $Q$ are written multiplicatively. 


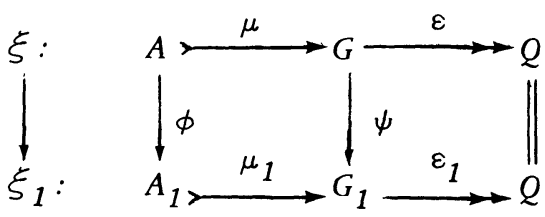

and we know [8]:

Proposition 3.3. Let $\phi: A \rightarrow A_{1}$ induce $\phi_{*}: H^{2}(Q ; A) \rightarrow H^{2}\left(Q ; A_{1}\right)$. Then $\phi_{*}(\xi)=\xi_{1}$.

Now let $\rho: Q^{\prime} \rightarrow Q$. Form the pull-back of $\rho$ and $\varepsilon(3.1)$; it is then known that we obtain a map of central extensions

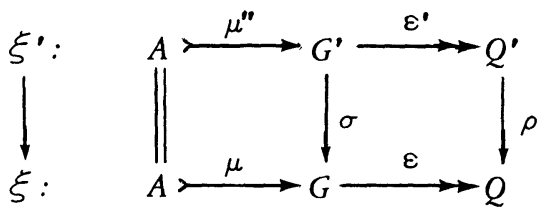

and we know $[8]$ :

PROPOSITION 3.4. Let $\rho: Q^{\prime} \rightarrow Q$ induce $\rho^{*}: H^{2}(Q ; A) \rightarrow H^{2}\left(Q^{\prime} ; A\right)$. Then $\rho^{*}(\xi)=\xi^{\prime}$. Moreover

$$
\phi_{*} \rho^{*}=\rho^{*} \phi_{*}
$$

PROPOSITION 3.5. Suppose given two central extensions

$$
\xi_{1}: A_{1} \stackrel{\mu_{1}}{\longrightarrow} G_{1} \stackrel{\varepsilon_{1}}{\longrightarrow} Q_{1}, \quad \xi_{2}: A_{2} \stackrel{\mu_{2}}{\longrightarrow} G_{2} \stackrel{\varepsilon_{2}}{\longrightarrow} Q_{2},
$$

and maps $\phi: A_{1} \rightarrow A_{2}, \rho: Q_{1} \rightarrow Q_{2}$. Then one may find $\tau: G_{1} \rightarrow G_{2}$ yielding a commutative diagram

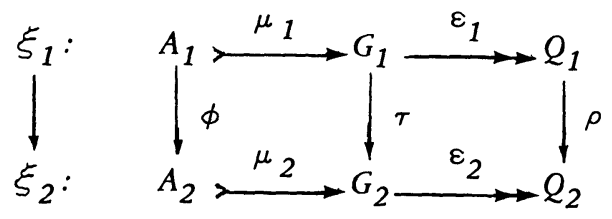

if and only if $\phi_{*}\left(\xi_{1}\right)=\rho^{*}\left(\xi_{2}\right)$. Moreover, if $\tau$ exists, then $\tau^{\prime}$ also yields a commutative diagram (3.6) if and only if

$$
\tau^{\prime}(x)=\tau(x) \mu_{2} \kappa \varepsilon_{1}(x), \quad x \in G_{1}, \text { for some } \kappa: Q_{1} \rightarrow A_{2} .
$$

PROOF. The key observation is that any diagram of the type (3.3), with common quotient group, must, in principle, have been constructed as (3.3) 
was constructed, so that the existence of the diagram implies that $\phi_{*}(\xi)=\xi_{1}$; and, similarly, any diagram of the type (3.4), with common kernel, must, in principle, have been constructed as (3.4) was constructed, so that the existence of the diagram implies that $\rho^{*}(\xi)=\xi^{\prime}$.

Now suppose that $\mathcal{\tau}$ exists in (3.6). Pulling back by means of $\rho$, we find a commutative diagram
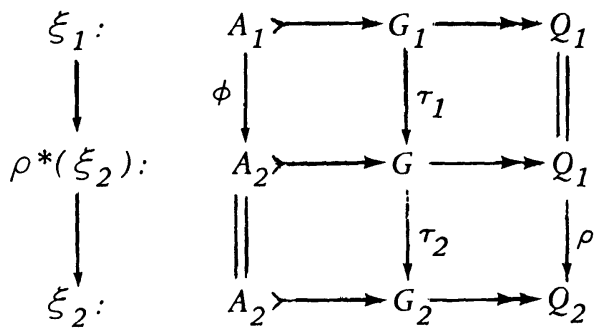

with $\tau_{2} \tau_{1}=\tau$. Thus, by the observation above, $\rho^{*}\left(\xi_{2}\right)=\phi_{*}\left(\xi_{1}\right)$.

Conversely, suppose that $\rho^{*}\left(\xi_{2}\right)=\phi_{*}\left(\xi_{1}\right)$. Then we have a diagram

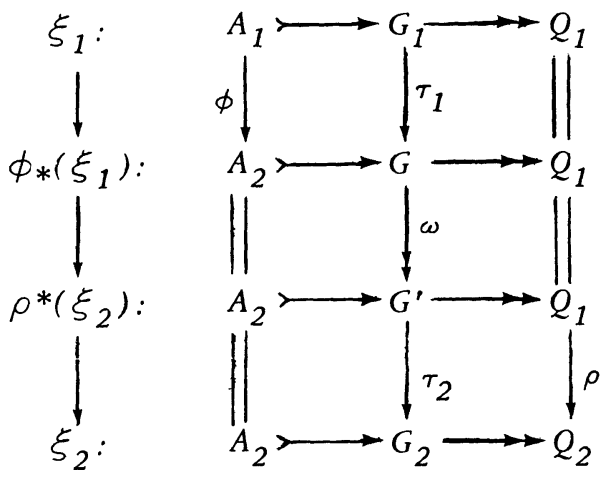

and we may take $\tau=\tau_{2} \omega \tau_{1}$.

The final statement of the proposition is almost immediate. Since $\varepsilon_{2} \tau=\varepsilon_{2} \tau^{\prime}$, we find a function $\theta: G_{1} \rightarrow A_{2}$, given by

$$
\mu_{2} \theta(x)=\tau(x)^{-1} \mathcal{T}^{\prime}(x), \quad x \in G_{1} .
$$

Moreover, $\theta$ is a homomorphism since $\xi_{2}$ is a central extension. Since

$$
\tau \mu_{1}=\tau^{\prime} \mu_{1}, \quad \theta \mu_{1}=0
$$

so $\theta$ induces $k: Q_{1} \rightarrow A_{2}$ with $k \varepsilon_{1}=\theta$. Conservely, if $\tau^{\prime}$ is given by 
(3.7), then $\tau^{\prime}$ is a homomorphism and preserves the commutativity of (3.6) when used to replace $\tau$.

PROPOSITION 3.6 (LYNDON-HOCHSCHILD-SERRE spectral sequence). Given any extension of groups

$$
M \longmapsto G \longrightarrow Q
$$

there exists a spectral sequence $\left\{E_{r}^{p q}\right\}$, with $E_{2}^{p q}=H_{p}\left(Q ; H_{q}^{M}\right)$, converging finitely to the graded group associated with $H_{*} G$, suitably filtered.

\section{Localization of nilpotent groups.}

Let $G$ be a group and let

$$
\cdots \subseteq \Gamma^{i+1}(G) \subseteq \Gamma^{i}(G) \subseteq \ldots
$$

be the lower central series of $G$; thus

$$
\Gamma^{1}(G)=G \text { and } \Gamma^{i+1}(G)=\left[G, \Gamma^{i}(G)\right] \text {. }
$$

We say that $G$ is nilpotent of class $c$ if

$$
\Gamma^{c}(G) \neq\{1\}, \quad \Gamma^{c+1}(G)=\{1\} .
$$

We then write $n i l G=c$.

DEFinition. Let $G$ be nilpotent. We say that $G$ is $P$-local if $x \mapsto x^{n}$, $x \in G$, is bijective for all $n \in P^{\prime}$. A homomorphism $e: G \rightarrow G_{P}$, with $G_{P}$ nilpotent, is said to $P$-localize $G$ if $G_{P}$ is $P$-local and $e$ has the universal property for homomorphisms of $G$ into $P$-local nilpotent groups: that is, if $H$ is $P$-local, then $e^{*}: \operatorname{Hom}\left(G_{P}, H\right) \cong \operatorname{Hom}(G, H)$.

DEFINITION. Let $G, H$ be nilpotent groups. We say that $\phi: G \rightarrow H$ is a $P$-isomorphism if:

(i) every element in $\operatorname{ker} \phi$ is a $P^{\prime}$-torsion element;

(ii) for every $y \in H$, there exist $n \in P^{\prime}, x \in G$, with $\phi(x)=y^{n}$.

It is immediately clear that this definition generalizes that given in 
Section 2 for abelian groups (nilpotent groups $G$ with nil $G \leqslant 1$ ). If $\phi$ satisfies (i) we call it $P$-injective, if $\phi$ satisfies (ii) we call it $P$-surjective.

Our main object in this section is to prove the following theorems.

THEOREM 4.1. With each nilpotent group $G$ we may associate its $P$-localization e: $G \rightarrow G_{P}$. Moreover nil $G_{P} \leqslant$ nil $G$.

THEOREM 4.2. A bomomorphism $\phi: G \rightarrow H$ of nilpotent groups $P$-localizes $G$ if and only if $H$ is $P$-local and $\phi$ is a $P$-isomorphism.

Let $N$ be the category of nilpotent groups, and let $N_{c}$ be the full subcategory of $N$ consisting of groups $G$ with nil $G \leqslant c$. We will achieve our objective by proving the following theorem by induction on $c$.

THEOREM 4.3. For each $c \geqslant 1$ we may find a functor $L_{c}: N_{c} \rightarrow N_{c}$ and $a$ natural transformation $e_{c}: 1 \rightarrow L_{c}$ such that $L_{c}(G)$ is $P$-local and $e_{c}(G)$, $G \in N_{c}$, has the universal property in $N_{c}$. We may choose $L_{c}, e_{c}$ so that

$$
L_{c}\left|N_{c-1}=L_{c-1}, \quad e_{c}\right| N_{c-1}=e_{c-1} .
$$

Further $\phi: G \rightarrow H \quad P$-localizes $G$ in $N_{c}$ if and only if $H$ is $P$-local and $\phi$ is a $P$-isomorphism.

PROOF. The assertion is true for $c=1$, for we constructed $L_{1}$ in Section 2 and proved Proposition 2.6. We now assume the assertion holds for $c-1$, $c \geqslant 2$, and prove it for $c$. We write $L$ for $L_{c-1}, e$ for $e_{c-1}$, and $G_{P}$ for $L G$. Thus we have defined

$$
L: N_{c-1} \rightarrow N_{c-1}, \quad e: 1 \rightarrow L,
$$

and wish to extend $L$ to $L: N_{c} \rightarrow N_{c}$, and to extend $e$ correspondingly, to have the universal property in $N_{c}$. We draw some consequences from the truth of Theorem 4.3 in the case $c-1$. First however we need to enunciate two propositions about $N$.

PROPOSITION 4.4. Let $G^{\prime} \stackrel{\mu}{\longrightarrow} G \stackrel{\varepsilon}{\longrightarrow} G^{\prime \prime}$ be a central extension in $N$. Then if $G^{\prime}, G^{\prime \prime}$ are $P$-local, so is $G$.

PROOF. Let $x \in G, n \in P^{\prime}$. Then $\varepsilon x=y^{n n}=\varepsilon y^{n}$, for some $y^{\prime \prime} \in G^{\prime \prime}, y \in G$. 
Thus $x=y^{n} \mu\left(y^{\prime}\right), y^{\prime} \in G^{\prime}$. But $y^{\prime}=x^{\prime n}$ for some $x^{\prime} \in G^{\prime}$, so

$$
x=y^{n} \mu\left(x^{\prime}\right)^{n}=\left(y \mu\left(x^{\prime}\right)\right)^{n},
$$

since $\mu G^{\prime}$ is central in $G$.

Suppose now that $x^{n}=y^{n}, x, y \in G, n \in P^{\prime}$. Then $\varepsilon x^{n}=\varepsilon y^{n}$, so

$$
\varepsilon x=\varepsilon y, \quad x=y \mu\left(x^{\prime}\right), \quad x^{\prime} \in G^{\prime} .
$$

Then $x^{n}=y^{n} \mu\left(x^{\prime n}\right)$, since $\mu G^{\prime}$ is central in $G$, so $x^{m n}=1, x^{\prime}=1, x=y$. PROPOSITION 4.5. Let

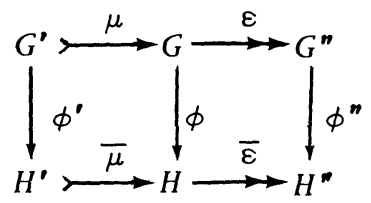

be a map of central extensions in $N$. Then if $\phi^{\prime}, \phi^{\prime \prime}$ are $P$-isomorphisms, so is $\phi$.

PROOF. Let $y \in H$. Since $\phi^{\prime \prime}$ is $P$-surjective, there exist $x^{n} \in G^{n}, n \in P^{\prime}$, with $\bar{\varepsilon}\left(y^{n}\right)=\phi "\left(x^{n}\right)$. Let $x^{n}=\varepsilon x_{0}, x_{0} \in G$. Then

$$
\bar{\varepsilon}\left(y^{n}\right)=\bar{\varepsilon} \phi\left(x_{0}\right) \text {, so } y^{n}=\phi\left(x_{0}\right) \bar{\mu}\left(y^{\prime}\right), \quad y^{\prime} \in H^{\prime} .
$$

Since $\phi^{\prime}$ is $P$-surjective, there exist $x^{\prime} \in G^{\prime}, m \in P^{\prime}$ with $y^{\prime m}=\phi^{\prime}\left(x^{\prime}\right)$. Then, since $\bar{\mu} H^{\prime}$ is central in $H$,

$$
y^{m n}=\phi\left(x_{0}^{m}\right) \bar{\mu}\left(y^{m}\right)=\phi\left(x_{0}^{m} \mu\left(x^{\prime}\right)\right), \quad m n \in P^{\prime},
$$

so $\phi$ is $P$-surjective.

Let $x \in G$ with $\phi x=1$. Then $\phi^{\prime \prime} \varepsilon(x)=1$ so, $\phi^{\prime \prime}$ being $P$-injective, there exists $n \in P^{\prime}$ with $\varepsilon\left(x^{n}\right)=1$. Thus $x^{n}=\mu x^{\prime}, x^{\prime} \in G^{\prime}$, and $\phi^{\prime} x^{\prime}=1$. Since $\phi^{\prime}$ is $P$-injective, $x^{\prime m}=1$ for some $m \in P^{\prime}$, so $x^{m n}=1$, $m n \in P^{\prime}$, and $\phi$ is $P$-injective.

We are now ready to exploit the inductive hypothesis to prove a series of propositions which will enable us to establish Theorem 4.3.

PROPOSITION 4.6. $L: N_{c-1} \rightarrow N_{c-1}$ is exact.

PROOF. We have the diagram, in $N_{c-1}$, 


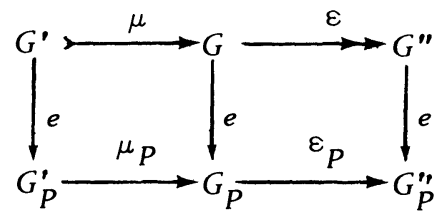

with the top row short exact, and wish to prove the bottom row short exact. We use the fact that $e$ is a $P$-isomorphism.

First, $\varepsilon_{P}$ is surjective. For let $y^{n} \in G_{P}^{m}$. Then, for some $n \in P^{\prime}$, $y^{m n}=e x^{n}, x^{n} \in G^{n}$. Let $x^{n}=\varepsilon x$. Then $y^{n n}=e \varepsilon x=\varepsilon_{P} e x$. Thus $\varepsilon_{P}$ is $P$-surjective, and one proves, just as in the proof of lemma 2.8 , that a $P$-surjection of $P$-local groups is a surjection.

Second, $\mu_{P}$ is injective. For let $\mu_{P} y^{\prime}=1, y^{\prime} \in G_{P}^{\prime}$. Then, for some $n \in P^{\prime}, y^{\prime n}=e x^{\prime}, x^{\prime} \in G^{\prime}$, so

$$
e \mu x^{\prime}=\mu_{P} e x^{\prime}=\mu_{P} y^{\prime n}=1 .
$$

Thus $\mu x^{\prime m}=1$ for some $m \in P^{\prime}$. Thus $x^{\prime m}=1, y^{\prime m n}=1$, and $m n \in P^{\prime}$, so $y^{\prime}=1$ since $G_{P}^{\prime}$ is $P$-local.

Third, $\mu_{P}$ is the kernel of $\varepsilon_{P}$. Of course $\varepsilon_{P} \mu_{P}=0$ so we must prove that $\operatorname{ker} \varepsilon_{P} \subset \operatorname{im} \mu_{P}$. Let $\varepsilon_{P} y=1, y \in G$. Then, for some $n \in P^{\prime}$, $y^{n}=e x, x \in G$, so

$$
e \varepsilon x=\varepsilon_{P} e x=\varepsilon_{P} y^{n}=1 \text {. }
$$

Thus $\varepsilon x^{m}=1$ for some $m \in P^{\prime}$. Thus $x^{m}=\mu x^{\prime}, x^{\prime} \in G^{\prime}$, so that $y^{m n}=$ $=\mu_{P} e x^{\prime}$, and $m n \in P^{\prime}$. Again one argues just as in the proof of Lemma 2.8 that, since $y^{m n} \in i m \mu_{P}$ and $G_{P}^{\prime}, G_{P}$ are $P$-local, therefore $y \in i m \mu_{P}$. PROPOSITION 4.7. If, in the commutative diagram, in $N_{c-1}$,

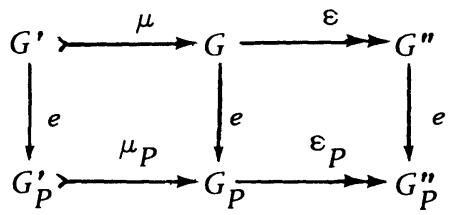

the top extension is central, so is the bottom.

PRoof. Let $x^{\prime} \in G^{\prime}, y \in G_{P}$. Then $y^{n}=e x, x \in G$, for some $n \in P^{\prime}$. Thus

$$
\left(\mu x^{\prime}\right)^{-1} x\left(\mu x^{\prime}\right)=x \text {, so }\left(\mu_{P} e x^{\prime}\right)^{-1} y^{n}\left(\mu_{P} e x^{\prime}\right)=y^{n} \text {. }
$$

Since $G_{P}$ has unique $n^{t h}$ roots, $\left(\mu_{P} e x^{\prime}\right)^{-1} y\left(\mu_{P} e x^{\prime}\right)=y$, so $\mu_{P} e x^{\prime}$ 
belongs to the center of $G_{P}$.

Now let $y^{\prime} \in G_{P}$. Then $y^{\prime m}=e x^{\prime}, x^{\prime} \in G^{\prime}$, for some $m \in P^{\prime}$. Thus, for any $y \in G_{P}$,

$$
y^{-1}\left(\mu_{P} y^{\prime}\right)^{m} y=\left(\mu_{P} y^{\prime}\right)^{m} .
$$

Since $G_{P}$ has unique $m^{\text {th }}$ roots, $y^{-1}\left(\mu_{P} y^{\prime}\right) y=\mu_{P} y^{\prime}$, so $\mu_{P} y^{\prime}$ belongs to the center of $G_{P}$. Thus $\mu_{P} G_{P}^{\prime}$ is central in $G_{P}$.

THEOREM 4.8. Let $G \in N_{i}, i \leqslant c-1$. Then $\widetilde{H}_{*}(e): \widetilde{H}_{*}(G) \rightarrow \widetilde{H}_{*}\left(G_{P}\right)$ $P$-localizes.

PROOF. We argue by induction on $i$, the theorem being true for $i=1$ (Proposition 2.5). Suppose the theorem true for all groups $K$ with nil $K \leqslant i-1$, $i \geqslant 2$, and let nil $G \leqslant i$. Let $Z$ be the center of $G$. Then nil $Z=1$, $n i l G / Z \leqslant i-1$, and, by Proposition 4.6 and 4.7, we have a map of central extensions

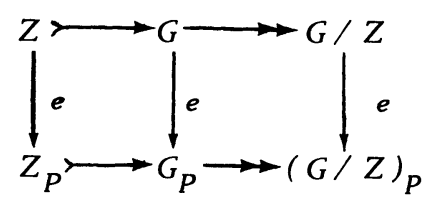

Then (4.3) induces a map of spectral sequences $\left\{E_{r}^{p q}\right\} \rightarrow\left\{\widetilde{E}_{r}^{p q}\right\}$, where (Proposition 3.6)

$$
E_{2}^{p q}=H_{p}\left(G / Z ; H_{q} Z\right), \quad \tilde{E}_{2}^{p q}=H_{p}\left((G / Z)_{P} ; H_{q} Z_{P}\right),
$$

the coefficients being trivial in both cases. It now follows from the inductive hypothesis, together with Propositions 2.3, 2.4, taken in conjonction with the natural universal coefficient sequence in homology, that (4.3) induces $e_{2}: E_{2}^{p q} \rightarrow \widetilde{E}_{2}^{p q}$ which is $P$-localization unless $(p, q)=(0,0)$. By Proposition 2.1 we now readily infer that $e_{\infty}: E_{\infty}^{p q} \rightarrow \widetilde{\sim}_{\infty}^{p q}$ is also $P$-localization unless $(p, q)=(0,0)$.

Since, for any $n, H_{n} G \quad\left(H_{n} G_{P}\right)$ has a finite filtration whose associated graded group is

$$
E_{\infty}^{p q} \quad\left(\tilde{E}_{\infty}^{p q}\right), \text { with } \quad p+q=n,
$$

it follows from Proposition 2.3 that $H_{n}(e): H_{n} G \rightarrow H_{n} G_{P} \quad P$-localizes for $n \geqslant 1$. 
COROLLARY 4.9. Let $G \in N_{c-1}$ and let $A$ be a $P$-local abelian group. Then $e: G \rightarrow G_{P}$ induces $e^{*}: H^{*}\left(G_{P} ; A\right) \cong H^{*}(G ; A)$.

PROOF. The case $n=0$ being trivial, we show that

$$
e^{*}: H^{n}\left(G_{P} ; A\right) \cong H^{n}(G ; A), \quad n \geqslant 1 .
$$

Consider the diagram

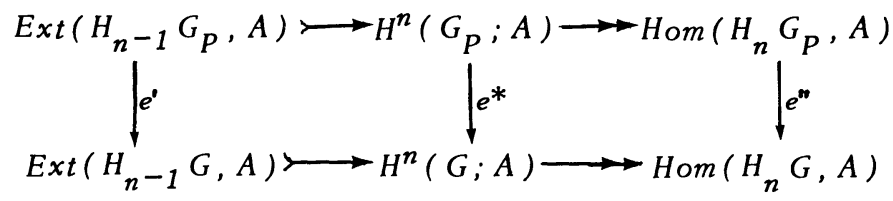

induced by $e$. It follows immediately from Theorem 4.8 and Proposition 2.9 that $e^{\prime \prime}$ is an isomorphism. If $n=1$ this completes the argument; if $n \geqslant 2$ it follows immediately from Theorem 4.8 and Proposition 2.9 that $e^{\prime}$ is an isomorphism. Thus $e^{*}$ is an isomorphism.

We are now ready to carry out the inductive step establishing Theorem 4.3 and hence Theorems 4.1, 4.2.

PROOF OF THEOREM 4.3. Assume $G$ nilpotent with nil $G \leqslant c$. We then have a central extension

$$
\Gamma^{c} \longleftrightarrow G \longrightarrow G / \Gamma^{c}, \quad \Gamma^{c}=\Gamma^{c}(G),
$$

with nil $\Gamma^{c} \leqslant 1$, nil $G / \Gamma^{c} \leqslant c-1$. Let (4.4) represent $\xi \in H^{2}\left(G / \Gamma^{c} ; \Gamma^{c}\right)$. Then $e_{*} \xi \in H^{2}\left(G / \Gamma^{c} ; \Gamma_{P}^{c}\right)$ and, by Corollary 4.9, there exists a unique element $\xi_{P} \in H^{2}\left(\left(G / \Gamma^{c}\right)_{P} ; \Gamma_{P}^{c}\right)$ such that

$$
e^{*} \xi_{P}=e_{*} \xi \text {. }
$$

Let the central extension

$$
\Gamma_{P}^{c} \longrightarrow G_{P} \longrightarrow\left(G / \Gamma^{c}\right)_{P}
$$

represent $\xi_{P}$. Then by Proposition 3.5 , we have a commutative diagram

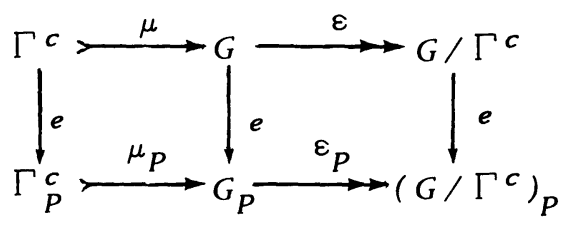

We first remark that, since (4.6) is a central extension and 
nill $\left.G / \Gamma^{c}\right)_{P} \leqslant c-1$, then $G_{P}$ is nilpotent and nil $G_{P} \leqslant c$. We next remark that, by Proposition 4.4, $G_{P}$ is $P$-local. We also remark that if, in fact, nil $G \leqslant c-1$, then

$$
\Gamma^{c}=\{1\}, \quad \Gamma_{P}^{c}=\{1\}, \quad G / \Gamma^{c}=G,
$$

and we naturally take $G_{P}=\left(G / \Gamma^{c}\right)_{P}$, preserving the same $P$-localization if we define $L G=G_{P}$, as we propose to do.

Now, by Proposition 4.5, e:G $\rightarrow G_{P}$ in (4.7) is a $P$-isomorphism. It follows, by a trivial modification of the argument of Lemma 2.8 (we restate the result in Lemma 4.11) that $e: G \rightarrow G_{P}$ is thus an isomorphism if $G$ is $P$-local. Then the universal property of $e$ will follow directly from the fact, still to be proved, that $e$ is a natural transformation of functors. For we then readily infer that

$$
e^{*}: \operatorname{Hom}\left(G_{P}, H\right) \rightarrow \operatorname{Hom}(G, H)
$$

is surjective if $H$ is $P$-local in $N_{c}$, and the fact that $e^{*}$ is injective follows immediately from the fact that $e$ is $P$-surjective and $H$ is $P$-local.

Thus it remains to define $L$ on morphisms of $N_{c}$ as a functor, and to prove the naturality of $e$. Let $\phi: G \rightarrow \bar{G}$ in $N_{c}$, and let $\bar{\Gamma}^{c}=\Gamma^{c}(\bar{G})$. Then we have

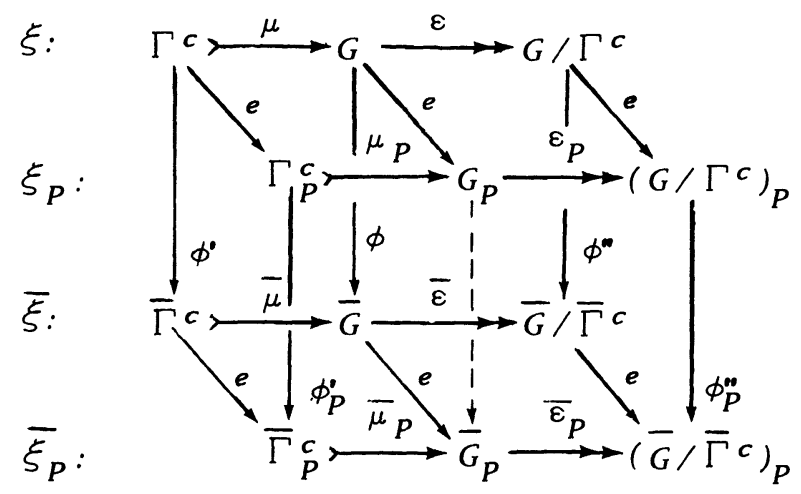

and our object is to define $\phi_{P}: G_{P} \rightarrow \bar{G}_{P}$ to make (4.8) commutative. It is clear that any $\phi_{P}$ yielding $\phi_{P} e=e_{\phi}$ is uniquely determined so the functoriality of $L$ is automatic once a suitable $\phi_{P}$ is defined.

In (4.8) $\phi^{\prime}, \phi^{\prime \prime}$ are induced by $\phi$, and

$$
e_{*} \xi=e^{*} \xi_{P}, \quad e_{*} \bar{\xi}=e^{*} \bar{\xi}_{P}
$$


Thus

$$
\begin{gathered}
e^{*} \phi_{P}^{m *} \bar{\xi}_{P}=\phi^{m *} e^{*} \bar{\xi}_{P}=\phi^{m *} e_{*} \bar{\xi}=e_{*} \phi^{m *} \bar{\xi}(3.5) \\
=e_{*} \phi_{*}^{*} \xi \text { (Prop. 3.5) }=\phi_{P}^{\prime} e_{*} \xi=\phi_{P}^{\prime} e^{*} \xi_{P}=e^{*} \phi_{P}^{\prime} \xi_{P}
\end{gathered}
$$

But

$$
e^{*}: H^{2}\left(\left(G / \Gamma^{c}\right)_{P} ; \bar{\Gamma}_{P}^{c}\right) \cong H^{2}\left(G / \Gamma^{c} ; \bar{\Gamma}_{P}^{c}\right),
$$

by Corollary 4.9, so $\phi_{P}^{m *} \bar{\xi}_{P}=\phi_{P}^{*} \xi_{P}$. Thus (Prop. 3.5) we may find $\tau: G_{P} \rightarrow \bar{G}_{P}$ so that

$$
\tau \mu_{P}=\bar{\mu}_{P} \phi_{P}^{\prime}, \quad \bar{\varepsilon}_{P} \tau=\phi_{P}^{\prime \prime} \varepsilon_{P} .
$$

Consider the diagram

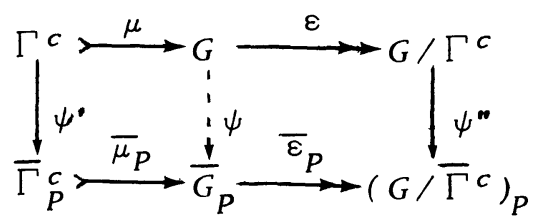

Here

$$
\psi^{\prime}=e \phi^{\prime}=\phi_{P}^{\prime} e, \quad \psi^{\prime \prime}=e \phi^{\prime \prime}=\phi_{P}^{m} e .
$$

It is thus clear that (4.10) commutes if $\psi=e \phi$ or $\psi=\tau e$, so that Proposition 3.5 implies that there is $\theta: G / \Gamma^{c} \rightarrow \bar{\Gamma}_{P}^{c}$, such that

$$
e \phi(x)=\tau e(x) \cdot \bar{\mu}_{P} \theta \varepsilon(x), \quad x \in G .
$$

Let $\theta_{P}:\left(G / \Gamma^{c}\right)_{P} \rightarrow \bar{\Gamma}_{P}^{c}$ be given by $\theta_{P} e=\theta$, and define

$$
\phi_{P}(y)=\tau(y) \cdot \bar{\mu}_{P} \theta_{P} \varepsilon_{P}(y), \quad y \in G_{P} \text {. }
$$

Again by Proposition 3.5 we have, from (4.9),

$$
\phi_{P} \mu_{P}=\bar{\mu}_{P} \phi_{P}^{\prime}, \quad \bar{\varepsilon}_{P} \phi_{P}=\phi_{P}^{\prime \prime} \varepsilon_{P}
$$

and also

$$
\phi_{P} e(x)=\tau e(x) \cdot \bar{\mu}_{P} \theta_{P} \varepsilon_{P} e(x)=\tau e(x) \cdot \bar{\mu}_{P} \theta \varepsilon(x)=e \phi(x), \quad x \in G .
$$

We are now left merely with the final assertion of Theorem 4.3. We know that $e: G \rightarrow G_{P}$ is a $P$-isomorphism and $G_{P}$ is $P$-local. We prove the converse just as for Proposition 2.6. We state the appropriate lemmas again, explicitly. 
LEMMA 4.10. If, in $N, \beta \alpha$ and $\alpha$ are $P$-isomorphisms, so is $\beta$.

PROOF. It is trivial that $\beta \alpha$ is $P$-surjective, so is $\beta$. Now consider $G_{1} \stackrel{a}{\longrightarrow} G_{2} \stackrel{\beta}{\longrightarrow} G_{3}$ and let $x_{2} \in G_{2}, \beta x_{2}=1$.

Since $\alpha$ is $P$-surjective, there exists $n$ prime to $P$ with $x_{2}^{n}=\alpha x_{1}, x_{1} \in G_{1}$. Then $\beta \alpha x_{1}=1$, so, $\beta \alpha$ being $P$-injective, there exists $m$ prime to $P$ with $x_{1}^{m}=1$. Then $x_{2}^{m n}=1, m n$ prime to $P$, so $\beta$ is $P$-injective.

LEMMA 4.11. In $N$ a $P$-isomorphism between $P$-local groups is an isomorphism.

PROOF. We make a trivial modification of the proof of Lemma 2.9 .

Now that the proof of Theorems 4.1, 4.2 is complete, we may, of course, restate Propositions 4.6, 4.7, Theorem 4.8 and Corollary 4.9 without any reference to the inductive parameter $c$. We ask the reader to assume those restatements made.

\section{Applications.}

In this section we show how the existence of a $P$-localization $e: G \rightarrow G_{P}$, together with the characterization of $e$ as a $P$-isomorphism to a $P$-local group, may be used to infer facts about nilpotent groups.

THEOREM 5.1. Let $\Pi$ be a family of primes. Then if $G$ is nilpotent it has a П-torsion subgroup $T_{\Pi}$.

P R Oof. Let $P=\Pi^{\prime}$. Localize at $P$ by $e: G \rightarrow G_{P}$. Since $e$ is a $P$-isomorphism, every element in kere is a $\Pi$-torsion element. Since $G_{P}$ is $P$-local, every $\Pi$-torsion element is in ker $e$. Thus ker $e=T_{\Pi}$.

THEOREM 5.2. Suppose $G$ is nilpotent and has no $\Pi$-torsion. Then if $x^{n}=y^{n}, x, y \in \Pi$, it follows that $x=y$.

PROOF. Localize at $P=\Pi^{\prime}$. Then $e: G \rightarrow G_{P}$ is injective and $e(x)^{n}=$ $=e(y)^{n}$. Since $G_{P}$ is $P$-local, $e(x)=e(y)$, so $x=y$. 
COROLLARY 5.3. The nilpotent group $G$ is $P$-local if and only if it has no $P^{\prime}$-torsion and $x \mapsto x^{n}, x \in G$, is surjective for all $n \in P^{\prime}$.

THEOREM 5.4. Let $G^{\prime} \longrightarrow G \rightarrow G^{\prime \prime}$ be a short exact sequence of nilpotent groups. Then if any two of $G^{\prime}, G, G^{\prime \prime}$ are $P$-local, so is the third.

PROOF. Since $P$-localization is exact, we obtain a map of short exact sequences

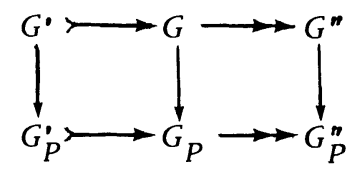

The hypothesis implies that two of the vertical arrows are isomorphisms. So therefore is the third, and the theorem is proved.

We now turn to results which make explicit mention of $P$-localization.

THEOREM 5.5. Let ,

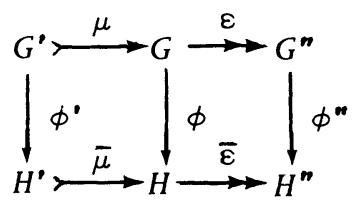

be a map of short exact sequences of nilpotent groups. Then if any two of $\phi^{\prime}, \phi, \phi^{\prime \prime} P$-localize, so does the third.

PROOF. By Theorem 5.4, we infer that $H^{\prime}, H, H^{\prime \prime}$ are all $P$-local. By $P$-localizing the top row, we obtain

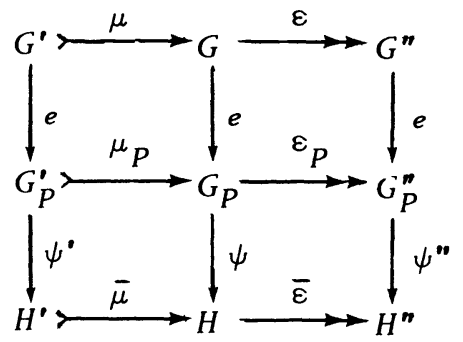

where $\psi^{\prime} e=\phi^{\prime}, \psi_{e}=\phi, \psi^{\prime \prime} e=\phi^{\prime \prime}$. Moreover, the commutativity relations 
$\bar{\mu} \psi^{\prime}=\psi \mu_{P}, \bar{\varepsilon} \psi=\psi^{\prime \prime} \varepsilon_{P}$ follow from the universal property of $e$. But the hypothesis implies that two of $\psi^{\prime}, \psi, \psi^{n}$ are isomorphisms. So therefore is the third, and the theorem is proved.

THEOREM 5.6. Let $G$ be nilpotent and let $\phi: G \rightarrow K P$-localize $G$. Then $\Gamma^{i} \phi: \Gamma^{i} G \rightarrow \Gamma^{i} K$ P-localizes $\Gamma^{i} G$, for all $i \geqslant 1$.

PROOF. It follows from Theorem 5.5 that it is sufficient to prove that the homomorphism $\phi_{i}: G / \Gamma^{i} G \rightarrow K / \Gamma^{i} K$, induced by $\phi, P$-localizes. We argue by induction on $i$, the assertion being trivial for $i=1$ and following from Theorem 4.8 for $i=2$. Thus we assume that $\phi_{i} P$-localizes, $i \geqslant 2$, and prove that $\phi_{i+1} P$-localizes. A second application of Theorem 5.5 shows that it is sufficient to prove that the homomorphism

$$
\bar{\phi}: \Gamma^{i} G / \Gamma^{i+1} G \rightarrow \Gamma^{i} K / \Gamma^{i+1} K,
$$

induced by $\phi, P$-localizes. We apply the 5 -term exact sequence in the homology of groups to the diagram

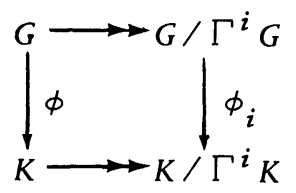

to obtain

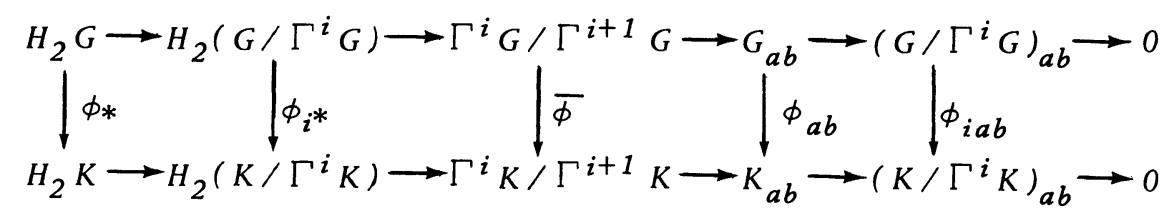

Then $\phi_{*}, \phi_{a b} P$-localize by Theorem 4.8 and $\phi_{i *}, \phi_{i a b} P$-localize by the inductive hypothesis and Theorem 4.8. It is now evident, by a mild extension of Proposition 2.3, that $\bar{\phi} P$-localizes.

Our next theorem appeared explicitly in [6]; we include it here for completeness.

THEOREM 5.7. Let $\phi: G \rightarrow K$ be a homomorphism of nilpotent groups. Then $\phi P$-localizes if and only if $\widetilde{H}_{*}(\phi): \widetilde{H}_{*}(G) \rightarrow \widetilde{H}_{*}(K) \quad P$-localizes. 
PRoOF. Theorem 4.8 asserts that $\widetilde{H}_{*}(\phi) P$-localizes if $\phi P$-localizes. We next prove that if $\widetilde{H}_{*}(K)$ is $P$-local, then $K$ is $P$-local. For let $e: K \rightarrow K_{P} \quad P$-localize. Then $\widetilde{H}_{*}(e): \widetilde{H}_{*}(K) \rightarrow \widetilde{H}_{*}\left(K_{P}\right) \quad P$-localizes; but $\widetilde{H}_{*}(K)$ is $P$-local, so $\widetilde{H}_{*}(e)$ is an isomorphism. It follows from the Stallings-Stammbach Theorem that $e$ is an isomorphism.

Now let $\widetilde{H}_{*}(\phi) P$-localize. Then $H_{*}(K)$ is $P$-local, so $K$ is $P$-local. Thus $\phi$ factors as $G \stackrel{e}{\longrightarrow} G_{P} \stackrel{\psi}{\longrightarrow} K$ and

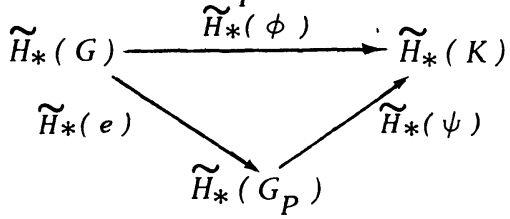

But since $\widetilde{H}_{*}(\phi), \widetilde{H}_{*}(e)$ both $P$-localize, $\widetilde{H}_{*}(\psi)$ is an isomorphism. Thus the Stallings-Stammbach Theorem again implies that $\psi$ is an isomorphism, so that $\phi P$-localizes.

We turn now to a consideration of the upper central series of a nilpotent group:

$$
Z(G)=Z^{1}(G) \subseteq Z^{2}(G) \subseteq \cdots \subseteq Z^{c}(G)=G,
$$

where $Z(G)$ is the center of $G$. We then easily prove

PROPOSITION 5.8. If $G$ is $P$-local, so is $Z^{i}(G), i \geqslant 1$. Moreover, $e: G \rightarrow G_{P}$ sends $Z^{i}(G)$ to $Z^{i}\left(G_{P}\right)$.

PRoOF. To prove $Z(G) P$-local, we only have to show that if $x \in G$ and $x^{n} \in Z(G), n \in P^{\prime}$, then $x \in Z(G)$. But if $x^{n} \in Z(G)$, then $y^{-1} x^{n} y=x^{n}$ for all $y \in G$. Taking $n^{t h}$ roots, $y^{-1} x y=x$, so $x \in Z(G)$. An easy induction, involving two applications of Theorem 5.4, now shows that $Z^{i}(G)$ is $P$-local, $i \geqslant 1$.

It was proved in Proposition 4.7 that $e: G \rightarrow G_{P}$ sends $Z(G)$ to $Z\left(G_{P}\right)$. Thus, again, an easy induction shows that $e$ sends $Z^{i}(G)$ to $Z^{i}\left(G_{P}\right), i \geqslant 1$.

Let us write $Z^{i}(e): Z^{i}(G) \rightarrow Z^{i}\left(G_{P}\right)$ for the restriction of $e$ : $G \rightarrow G_{P}$. Then we may prove 
THEOREM 5.9. If $G$ is finitely generated nilpotent and $e: G \rightarrow G_{P} P$-localizes $G$, then the restriction $Z^{i}(e): Z^{i}(G) \rightarrow Z^{i}\left(G_{P}\right) \quad P$-localizes $Z^{i}(G)$.

PROOF. This was proved in [6] for $i=1$, but we will repeat the proof in order to demonstrate that only facts about nilpotent groups explicitly mentioned in this paper are required. It is plain, in view of Proposition 5.8 and Theorem 4.2 , that we only have to prove that $Z(e)$ is $P$-surjective. We proceed by a series of lemmas.

LEMMA 5.10. Let $G$ be a group, $x, y \in G$ and the commutator $[x, y] \epsilon \Gamma^{i}$. Then $\left[x, y^{n}\right] \equiv[x, y]^{n} \bmod \Gamma^{i+1}$.

PROOF. This is immediately evident from the commutator identity

$$
\left[x, y_{1} y_{2}\right]=\left[x, y_{1}\right]\left[y_{1},\left[x, y_{2}\right]\right]\left[x, y_{2}\right] \text {, }
$$

where $[x, y]=x y x^{-1} y^{-1}$.

LEMMA 5.11. Let $G$ be nilpotent and $[x, y] \in T_{\Pi}$, the $\Pi$-torsion subgroup of $G$ (Theorem 5.1). Then $\left[x, y^{n}\right]=1$ for some $n \in \Pi$.

PROOF. First observe (by passing to the quotient group $G / T_{\Pi}$ ) that if $[x, y] \epsilon T_{\Pi}$, then $\left[x, y^{r}\right] \in T_{\Pi}$ for all $r$. Now, since $G$ is nilpotent, it suffices to show that, for each $i$, there exists $n_{i} \in \Pi$ with $\left[x, y^{n_{i}}\right] \epsilon \Gamma^{i}$. For $i=2$ take $n_{i}=1$, and proceed by induction on $i$. For if $\left[x, y^{n_{i}}\right] \in \Gamma^{i}$, $n_{i} \in \Pi$, then, since $\left[x, y^{n_{i}}\right] \in T_{\Pi}$, there exists $m_{i} \in \Pi$ with $\left[x, y^{n_{i}}\right]^{m_{i}}=$ $=1$, and, by Lemma 5.10,

$$
\left[x, y^{n_{i} m_{i}}\right] \in \Gamma^{i+1}, \quad n_{i} m_{i} \in \Pi .
$$

LEMMA 5.12. Let $G$ be finitely generated nilpotent, and let $e(y) \in Z\left(G_{P}\right)$. Then there exists $n \in P^{\prime}$ with $y^{n} \in Z(G)$.

PROOF. Let $G=\left(x_{1}, x_{2}, \ldots, x_{k}\right)$. For each $x_{j}$ we have $e\left[x_{j}, y\right]=1$, so that $\left[x_{j}, y\right] \in T_{P^{\prime}}$. Thus there exists $n_{j} \in P^{\prime}$, with $\left[x_{j}, y^{n_{j}}\right]=1$, by Lemma 5.11. Set $n=n_{1} n_{2} \ldots n_{k}$. Then

$$
n \in P^{\prime} \quad \text { and } \quad\left[x_{j}, y^{n}\right]=1, \quad j=1,2, \ldots, k \text {, }
$$

so that $y^{n} \in Z(G)$. 
We now prove that $Z(e): Z(G) \rightarrow Z\left(G_{P}\right)$ is $P$-surjective. Let $b \in Z\left(G_{P}\right)$. Then there exists $m \in P^{\prime}$ with $b^{m}=e(y), y \in G$. By Lemma 5.12, there exists $n \in P^{\prime}$ with $y^{n} \in Z(G)$ and $e\left(y^{n}\right)=b^{m n}, m n \in P^{\prime}$. Thus $Z(e)$ is $P$-surjective and Theorem 5.9 is proved for $i=1$.

The argument is now completed by an easy induction. Assume $i \geqslant 2$, and that $Z^{i-1}(e) P$-localizes. Then the induced map

$$
e_{i}: G / Z^{i-1}(G) \rightarrow G_{P} / Z^{i-1}\left(G_{P}\right)
$$

also $P$-localizes, by Theorem 5.5. Moreover, $G / Z^{i-1}(G)$ is finitely generated, so that

$$
Z\left(e_{i}\right): Z^{i}(G) / Z^{i-1}(G) \rightarrow Z^{i}\left(G_{P}\right) / Z^{i-1}\left(G_{P}\right)
$$

$P$-localizes. Finally, a second application of Theorem 5.5 establishes that $Z^{i}(e) P$-localizes.

\section{P-isomorphisms.}

It is trivial to prove that a composite of $P$-isomorphisms is a $P$-isomorphism. It thus readily follows from Lemmas $4.10,4.11$ that if, in the diagram

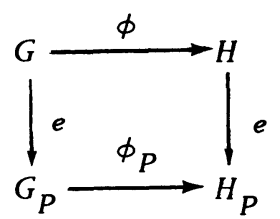

in $N, \phi$ is a $P$-isomorphism, then $\phi_{P}$ is an isomorphism. Our main objective in this section is to prove the converse of this, and thereby to be able to obtain consequences about the properties of $P$-isomorphisms of nilpotent groups. However, the converse appears to require deeper properties of nilpotent groups than those so far exploited. We will use Philip Hall's theory of basic commutators [5] to prove the following result, which we have not succeeded in finding in the literature.

THEOREM 6.1. Let $G$ be a group, $x, y \in G$ with $y^{n}=1$. Then

$$
(x y)^{n^{i}} \equiv x^{n^{i}} \bmod \Gamma^{i+1}(G) .
$$


PROOF. We may assume without loss of generality that $G$ is generated by $x, y$. Then $\Gamma^{i}(G) / \Gamma^{i+1}(G)$ is generated by the cosets of certain $i$-fold basic commutators. Moreover, an $i$-fold commutator is linear in each of its arguments, modulo $\Gamma^{i+1}(G)$. Since an $i$-fold basic commutator, $i \geqslant 2$, must involve $y$ as an argument, it follows that, for any such commutator $c$, $c^{n} \in \Gamma^{i+1}(G)$, so that, for any element

$$
q \in \Gamma^{i}(G), \quad i \geqslant 2, \quad q^{n} \in \Gamma^{i+1}(G) \text {. }
$$

We now proceed to prove the theorem by induction on $i$. If $i=1$, then

$$
(x y)^{n} \equiv x^{n} y^{n} \bmod \Gamma^{2}=x^{n} .
$$

Now suppose that $(x y)^{n^{i}}=x^{n^{i}} q$, for some $i \geqslant 1$, with $q \in \Gamma^{i+1}$. Then $q^{n} \in \Gamma^{i+2}$, so

$$
(x y)^{n^{i+1}}=\left(x^{n^{i}} q\right)^{n} \equiv x^{n^{i+1}} q^{n} \bmod \Gamma^{i+2} \equiv x^{n^{i+1}} \bmod \Gamma^{i+2},
$$

and the theorem is proved.

COROLLARY 6.2. Let $G$ be a nilpotent group with nil $G \leqslant c$. Then if $x$, $y \in G$, with $y^{n}=1$,

$$
(x y)^{n^{c}}=x^{n^{c}} \text {. }
$$

PROPOSITION 6.3. Given $G_{1} \stackrel{a}{\longrightarrow} G_{2} \stackrel{\beta}{\longrightarrow} G_{3}$ in $N$, then if $\beta$ and $\beta$ are $P$-isomorphisms, so is $\alpha$.

PROOF. It is trivial that $\alpha$ is $P$-injective if $\beta \alpha$ is $P$-injective. Now let $y \in G_{2}$. Since $\beta a$ is $P$-surjective, $\beta\left(y^{m}\right)=\beta \alpha(x)$, for some $x \in G_{1}$, $m \in P^{\prime}$. Then, since $\beta$ is $P$-injective, $y^{m}=\alpha(x) b$, with $b \in G_{2}$ and $b^{n}=1$, $n \in P^{\prime}$. By Corollary 6.2,

$$
y^{m n^{c}}=\alpha\left(x^{n^{c}}\right) \quad \text { where } \quad n i l G_{2} \leqslant c .
$$

Since $m n^{c} \in P^{\prime}$, it follows that $\alpha$ is $P$-surjective.

COROLLARY 6.4. In the diagram (6.1) in $N, \phi$ is a $P$-isomorphism if and only if $\phi_{P}$ is an isomorphism.

It now follows (see [4]) that the $P$-isomorphisms of $N$ are pre- 
cisely those morphisms of $N$ which are rendered invertible by the localization functor; we are thus led to the general theory of completions as in $[3,4]$, but we do not take that direction here. Instead, we draw some immediate consequences.

THEOREM 6.5. Let

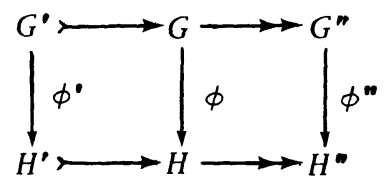

be a map of short exact sequences of nilpotent groups. Then if any two of $\phi^{\prime}, \phi, \phi^{\prime \prime}$ are $P$-isomorphisms, so is the third.

PROOF. We $P$-localize. Since $P$-localization is exact and two of $\phi_{\dot{P}}$, $\phi_{P}, \phi_{P}^{\prime \prime}$ are isomorphisms, so is the third. The theorem thus follows from Corollary 6.4 .

THEOREM 6.6. Let $\phi: G \rightarrow K$ be a $P$-isomorphism of nilpotent groups. Then $\Gamma^{i} \phi: \Gamma^{i} G \rightarrow \Gamma^{i} K$ is a $P$-isomorphism.

Proof. We $P$-localize. Since $\phi_{P}$ is an isomorphism $\Gamma^{i}\left(\phi_{P}\right)$ is an isomorphism. But, by Theorem 5.6, $\Gamma^{i}\left(\phi_{P}\right)=\left(\Gamma^{i} \phi\right)_{P}$. Thus, by Corollary $6.4, \Gamma^{i} \phi$ is a $P$-isomorphism.

THEOREM 6.7. Let $\phi: G \rightarrow K$ be a homomorphism of nilpotent groups. Then $\phi$ is a $P$-isomorphism if and only if $\widetilde{H}_{*}(\phi): \widetilde{H}_{*}(G) \rightarrow \widetilde{H}_{*}(K)$ is a $P$-isomorphism.

PRoOF. We $P$-localize. Then:

$\phi$ is a $P$-isomorphism $\Leftrightarrow \phi_{P}$ is an isomorphism $\Leftrightarrow \widetilde{H}_{*}\left(\phi_{P}\right)$ is an isomorphism $\Leftrightarrow \widetilde{H}_{*}(\phi)_{P}$ is an isomorphism $\Leftrightarrow \widetilde{H}_{*}(\phi)$ is a $P$-isomorphism.

Here the first equivalence uses Corollary 6.4 ; the second uses the StallingsStammbach Theorem; the third uses Theorem 5.7; and the fourth again uses Corollary 6.4 .

THEOREM 6.8. The following assertions about the nilpotent groups $G, K$ 
are equivalent (see [4]):

(i) $G_{P}$ and $K_{P}$ are isomorpbic;

(ii) there exists a nilpotent group $L$ and $P$-isomorpbisms $\alpha: G \rightarrow L$, $\beta \cdot K \rightarrow L$;

(iii) there exists a nilpotent group $M$ and $P$-isomorphisms $\gamma: M \rightarrow G$, $\delta: M \rightarrow K$.

PROOF. It is plain from Corollary 6.4 that (ii) $\Rightarrow$ (i), (iii) $\Rightarrow$ (i). Also, since $e$ is a $P$-isomorphism, the diagram

$$
G \stackrel{\omega e}{\longrightarrow} K_{P} \stackrel{e}{\longleftarrow} K, \text { where } \omega: G_{P} \cong K_{P},
$$

shows that (i) $\Rightarrow$ (ii). That (ii) $\Rightarrow$ (iii) now follows from

PROPOSITION 6.9. Let

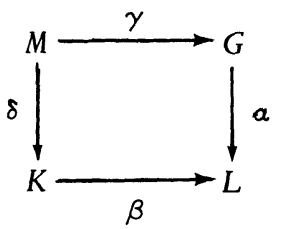

be a pull-back in the category of groups, and let $G, K, L$ be nilpotent. Then $M$ is nilpotent and $\delta$ is a $P$-isomorphism if $a$ is a $P$-isomorphism. PROOF. $M$ is a subgroup of $G \times K$ and thus certainly nilpotent. Since ker $\alpha \cong k e r \delta$ it is plain that $\delta$ is $P$-injective if and only if $\alpha$ is $P$-injective. Now let $\alpha$ be $P$-surjective and let $x \in K$. Then there exists $y \in G$, $n \in P^{\prime}$ with $\alpha(y)=\beta\left(x^{n}\right)$. But then

$$
\left(y, x^{n}\right) \in M \quad \text { and } \quad \delta\left(y, x^{n}\right)=x^{n},
$$

so $\delta$ is $P$-surjective. This proves Proposition 6.9 and, with it, Theorem 6.8 .

Let us call $G, K P$-isomorphic if any of conditions (i), (ii), (iii) of Theorem 6.8 hold.

THEOREM 6.10. Let $G, K$ be finitely generated $P$-isomorphic nilpotent groups. Then $Z^{i}(G), Z^{i}(K)$ are $P$-isomorphic for all $i \geqslant 1$.

P R oof. Apply Theorem 5.9.

REMARK. In fact, in the category of nilpotent groups, the four implications 


$$
\phi P \text {-inj } \Longleftrightarrow \phi_{P} \text { inj, } \phi P \text {-surj } \Longleftrightarrow \phi_{P} \text { surj }
$$

are all valid, refining Corollary 6.4 . Of these, the implication

$$
\phi_{P} \text { surj } \Rightarrow \phi P \text {-surj }
$$

alone requires Corollary 6.2, the others being elementary. Using these implications, one may obtain the following variant of the Stallings-Stammbach Theorem:

THEOREM 6.11. Let $\phi: G \rightarrow K$ be a bomomorphism of nilpotent groups. Then $\phi$ is a $P$-isomorphism if and only if $\phi_{a b}$ is a $P$-isomorphism and $\mathrm{H}_{2}(\phi)$ is a $P$-surjection.

\section{Localization of finitely generated nilpotent groups.}

In this section we prove three theorems which are useful in applications of localization to homotopy theory. We begin with some lemmas.

LEMMA 7.1. [5] A subgroup of a finitely generated nilpotent group is finitely generated.

PROOF. From the Hall theory of basic commutators and an easy induction we readily infer that if $G$ is finitely generated nilpotent, so are the terms $\Gamma^{i} G$ of the lower central series. We now prove the lemma by induction on nil $G$, since it is true if $n i l G=1$.

Let $H$ be a subgroup of $G$. Then $H \cap \Gamma^{2} G$ is a subgroup of $\Gamma^{2} G$ and $n i l \Gamma^{2} G<n i l G$, so, by the inductive hypothesis, $H \cap \Gamma^{2} G$ is finitely generated. We have the short exact sequence

$$
H \cap \Gamma^{2} G \longrightarrow H \longrightarrow H \Gamma^{2}(G) / \Gamma^{2}(G)
$$

but $H \Gamma^{2}(G) / \Gamma^{2}(G) \subseteq G_{a b}$, and hence is finitely generated. Thus $H$ is itself finitely generated.

Let $P_{l}, l \geqslant 0$, be the complement of the set $\left\{p_{1}, p_{2}, \ldots, p_{l}\right\}$ consisting of the first $l$ primes. 
L EMMA 7.2. If $G$ is finitely generated nilpotent, then there exists $l$ such that $G_{P_{l}} \rightarrow G_{0}$ is injective, where $G_{0}$ is the rationalization of $G$.

PROOF. Since $G$ is finitely generated, so is its torsion subgroup $T$. Since $T$ is then easily seen to be finite, $T$ has $p$-torsion for only finitely many primes $p$. Thus, for $l$ sufficiently large, $G_{P_{l}}$ is torsion free and $G_{P_{l}} \rightarrow G_{0}$ is injective.

THEOREM 7.3. Let $G, K$ be finitely generated, nilpotent groups and let $\phi: G \rightarrow K_{0}$ be a bomomorphism. Then there exists $l$ such that $\phi$ has a unique lift into $K_{P_{l}}$.

PROOF. We first choose $l$ so that $K_{P_{l}} \rightarrow K_{0}$ is injective. Let $\left(x_{1}, x_{2}, \ldots, x_{n}\right)$ generate $G$, let $e: K \rightarrow K_{0}$ rationalize $K$, and then find $y_{i} \in K, m_{i} \in \mathbf{N}$, such that

$$
e\left(y_{i}\right)=\phi\left(x_{i}\right)^{m}, \quad i=1,2, \ldots, n .
$$

Now subject $l$ to the further condition that

$$
m_{i} \in P_{l}^{\prime}, \quad i=1,2, \ldots, n,
$$

and factorize $e$ as

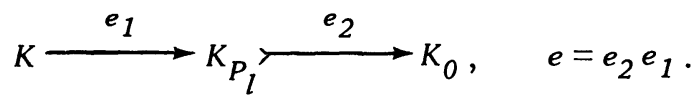

Since $K_{P_{l}}$ is $P_{l}$-local, we have

$$
\begin{array}{cl}
e_{1}\left(y_{i}\right)=z_{i}^{m_{i}}, \quad \text { so } & \phi\left(x_{i}\right)^{m_{i}}=e_{2}\left(z_{i}\right)^{m_{i}}, \\
\phi\left(x_{i}\right)=e_{2}\left(z_{i}\right), & i=1,2, \ldots, n,
\end{array}
$$

and $\phi$ lifts uniquely into $K_{P_{l}}$.

Let us say that the nilpotent group $G$ has property $A$ if $G$ is the pull-back of its localizations $G_{P}$ over its rationalization $G_{0}$. Then it has been observed (e.g. in [6]) that a finitely generated abelian group has property A.

PROPOSITION 7.4. Let $G^{\prime} \stackrel{\mu}{\longrightarrow} G \stackrel{\varepsilon}{\longrightarrow} G^{\prime \prime}$ be a short exact sequence of nilpotent groups. Then if $G^{\prime}, G^{\prime \prime}$ have property A, so bas $G$.

PROOF. Write $e_{p}: G \rightarrow G_{p}$ for the localization, $r_{p}: G_{p} \rightarrow G_{0}$ for the rationa- 
lization. We want to prove that, given $g_{p} \in G_{p}$, with $r_{p} g_{p}=g_{0}$, for all $p$, there exists a unique $g \in G$ with $e_{p} g=g_{p}$. Now

$$
\varepsilon_{p} g_{p} \in G_{p}^{\prime \prime} \quad \text { and } \quad r_{p}^{\prime \prime} \varepsilon_{p} g_{p}=\varepsilon_{0} g_{0}
$$

for all $p$. Since $G^{\prime \prime}$ has property $A$, there exists a unique $g^{\prime \prime} \in G^{n}$ with $e_{p}^{\prime \prime} g^{\prime \prime}=\varepsilon_{p} g_{p}$. Let $g^{\prime \prime}=\varepsilon \bar{g}$. Then $\varepsilon_{p} g_{p}=\varepsilon_{p} e_{p} \bar{g}$, so that $g_{p}=\left(e_{p} \bar{g}\right) g_{p}^{\prime}$, where $g_{p}^{\prime} \in G_{p}^{\prime}$, and we regard $\mu, \mu_{p}, \mu_{0}$ as inclusions. Moreover $g_{0}=(r \bar{g})\left(r_{p}^{\prime} g_{p}^{\prime}\right)$ for all $p$, where $r: G \rightarrow G_{0}$ is the rationalization, so that the elements $g_{p}^{\prime}$ have a common rationalization. Since $G^{\prime}$ has property $A$, there exists a unique $g^{\prime} \in G^{\prime}$ with $e_{p}^{\prime} g^{\prime}=g_{p}^{\prime}$. Then $g_{p}=e_{p}\left(\vec{g} g^{\prime}\right)$.

It remains to prove uniqueness. We suppose $g \in G$ with $e_{p} g=1$, for all $p$. Then $e_{p}^{\prime \prime} \varepsilon g=1$, for all $p$, so $\varepsilon g=1$, by uniqueness, and $g \in G^{\prime}$. But then $e_{p}^{\prime} g=1$, for all $g$, so $g=1$ by uniqueness.

THEOREM 7.5. A finitely generated nilpotent group bas property A.

PROOF. We argue by induction on the nilpotency class of the group $G$, since the theorem is true if nil $G=1$. We suppose nil $G=c \geqslant 2$, and that the theorem has been proved for finitely generated nilpotent groups of class $\leqslant c-1$. But then (see Lemma 7.1)

$$
\Gamma^{2}(G) \longleftrightarrow G \longrightarrow G / \Gamma^{2}(G)
$$

is a short exact sequence of finitely generated nilpotent groups with nil $\Gamma^{2} G<$ nil $G, \quad n i l G / \Gamma^{2} G=1$.

Thus $\Gamma^{2} G$ and $G / \Gamma^{2} G$ have property $A$ and so, by Proposition 7.4, has $G$.

THEOREM 7.6. Let $\phi: G \rightarrow H$ be a bomomorphism of nilpotent groups. Then $\phi$ is an isomorphism if and only if $\phi_{p}$ is an isomorphism for all $p$.

PROOF. We assume $\phi_{p}$ an isomorphism for all $p$, that is, $\phi$ is a $p$-isomorphism for all $p$. Since $k e r \phi$ is a torsion group, and all primes are forbidden, $\operatorname{ker} \phi=\{1\}$. Now let $y \in H$. Then, for each $p$, we have $x_{(p)} \in G$, $n_{p}$ prime to $p$, and $y^{n} p=\phi x_{(p)}$. Since $\operatorname{gcd}\left(n_{p}\right)=1$, we may findintegers $a_{p}$, almost all zero, such that $\Sigma a_{p} n_{p}=1$. Set $x=\Pi \underset{x}{a p}(p)$. It is then plain that $y=\phi x$. 


\section{Bibliography.}

1. G. BAUMSLAG, Lecture Notes on Nilpotent Groups, A.M.S. Regional Conference Series No. 2 (1971).

2. G. BAUMSLAG, Some remarks on nilpotent groups with roots, Proc. Amer. Math. Soc. 12 (1961), 262-267.

3. A. K. BOUSFIELD and D. M. KAN, Homotopy limits, completions and localizations, Lecture Notes in Mathematics 304, Spinger Verlag (1972).

4. A. DELEANU, A. FREI and P. HILTON, Generalized Adams completion (to appear).

5. P. HALL, Edmonton notes on nilpotent groups, Queen Mary College Mathematical Notes (1969).

6. P. HILTON, G. MISLIN and J. ROITBERG, Homotopical localization, Proc. Lond. Math. Soc. 26 (1973) (to appear).

7. P. HILTON, G. MISLIN and J. ROITBERG, Topological localization and nilpotent groups, Bull. A.M.S. (1973) (to appear).

8. P. HILTON and U. STAMmBACH, A Course in Homological Algebra, Graduate Texts in Mathematics, No. 4, Springer Verlag (1971).

9. A. G. KUROsH, The Theory of Groups, Vol. 2, Chelsea Publishing Company (1960).

10. M. LAZARD, Sur les groupes nilpotents et les anneaux de Lie, Ann. Sci. Ecole Norm. Sup. 71 (1954), 101-190.

11. R. B. WARFIELD, Jr., Localization of nilpotent groups, University of Washington (1972) (mimeographed).

Battelle Seattle Research Center

Case Western Reserve University

University of Washington 